\section{CBT of a Person Living in a Situation of Mental Handicap and Presenting an Anxiety Disorder Coupled with a Specific Phobia}

\section{Abstract}

The prevalence of anxiety disorders within a population presenting an intellectual deficiency is $43 \%$. The integrated multimodal approach highlights different predisposing risk factors: vulnerability and its consequences on adaptive behaviors and deficits in the learning process; interactions with the environment. Negative experiences and a weak perception of control lead to a higher failure expectation, resulting in an increase in anxiety for the handicapped person. On the other hand, environment, family and professionals may induce responses of dependency, fear and avoidance, and consequently, a decrease of opportunities to develop social and emotional skills. The cognitive-behavioral therapy was adapted to the patient. The therapeutic alliance with the patient, as well as with his family and professionals, was the keystone and the required condition for the therapy procedure.

The functional analysis underlined three therapeutic axes: 1) a work on the physiological reactions of anxiety and the train phobia, using the reaction management techniques

2) A work on cognitive restructuration.

3) A work on the behavior during exposure to the anxiety-inducing situation. Simultaneously, the patient received an antidepressant and anti-anxiety medication.

Other skills and techniques were used: eye tracking (ASL mobile) to point out the patient resources; the psycho-educative approach using the "heart mat" (cardiac coherence) as a mediator to share the understanding of the situation; a motivational approach for the exposure planning; and the PEUR model, used in group therapies to divide the therapy into sequences, thus fostering self-control. Measurements at frequent and regular intervals have shown the rapid evolution of the patient and the pertinence of the cognitive-behavioral approach during therapies for persons living in a situation of mental handicap and presenting concomitant mental disorders.

Keywords: Mental handicap; Anxiety; Intellectual disabilities

\section{Pierre El korh and Fabienne Giuliani}

Psychiatry and Mental Development Department (Community Psychiatry Service, Psychiatry Department, CHUV, Switzerland

Corresponding author: Fabienne Giuliani

\section{$\equiv$ Pierre.El-Korh@chuv.ch \\ Fabienne.Giuliani@chuv.ch}

Psychiatry and Mental Development Department,Community Psychiatry Service, Psychiatry Department, CHUV, Switzerland

\section{Tel: 41213142185}

Citation: El korh P, Giuliani F. CBT of a Person Living in a Situation of Mental Handicap and Presenting an Anxiety Disorder Coupled With a Specific Phobia. 2016, 2:2.

Received: April 07, 2016; Accepted: April 23, 2016; Published: April 28, 2016

\section{Introduction}

The theoretical context of the problem and the prevalence of anxiety disorders in a population of people with intellectual disabilities (ID)

Epidemiological studies show that generalized anxiety and specific phobias are the most common anxiety disorders in people with I.D., with lifetime prevalences of 5 to $11.3 \%$ [1,2].

And if Essau [3] indicates a higher probability of developing a psychopathology in children and adolescents with I.D. compared to those without, Dekker and Koot $[4,5]$ cite a prevalence of $21.9 \%$. 
The prevalence of anxiety disorders in the population of people with I.D. having a severe mental handicap is $43 \%$ [6].

\section{Predisposing risk factors}

Despite the similarity that exists between risk factors in this population and those without ID [7] intellectual disability is a risk factor for the development of anxiety disorders and has harmful consequences on the development of adaptive behaviours [8]. Individuals with an I.D. would also have a biological vulnerability to psychopathological disorders caused, for example, by the pre or postnatal condition having caused the ID. Furthermore, the ID leads to shortfalls in the learning process. Negative experiences, associated with a weak perception of control, lead to a higher expectation of failure which then increases anxiety. Difficulties with social adaptation and managing environmental stress make it so that people with an ID respond to stressful situations with an elevated level of anxiety [9].

Also, when a child's cognitive development doesn't conform to normal development, parents may become anxious. And thus, through imitation, the child may develop anxiety [8]. Family members and professionals also tend to infantilize and overprotect those with intellectual disabilities, something which leads to responses of dependency, fear, and avoidance $[8,10]$. This then means that individuals with ID have fewer opportunities to develop emotional management skills compared to those without.

Logically, other risk factors, protection mechanisms, comorbidities and the consequences of anxiety disorders for the general population also apply to those with cognitive impairments. In terms of developmental trajectory, anxiety is more diffuse and lasts longer in the I.D. population compared to the general population [8].

\section{Intervention}

\section{Context of the request}

Mr. JR's socio-professional advisor, in the training and improvement centre of a socio-educational institute, requested an intervention. A meeting with Mr. JR, his parents, and his advisors then made this request formal.

Upon his return from vacation, a month earlier, Mr. JR developed severe anxiety which manifested itself mainly as an agoraphobia without a panic disorder (CIM-10 : F300.01). Mr. JR avoided taking the train, which meant his Home - Institution - Home travel had to done using a taxi. It's important to note that during the preceding vacation, Mr. JR spoke of suicide and "scratched" his stomach with a knife. He was treated by a family doctor, who prescribed Lexotanil.

\section{First meeting}

Mr. JR is a young man of average height, well-dressed, wearing a hat, glasses and hearing aids. Although hesitant, he is very aware of his difficulties and expresses them clearly. He explained to us the appearance of this anxiety during a visit to the bear pit in a zoo. Since then, he has been struck with severe anxiety and a phobia of taking the train alone. He spoke to us of the treatments he received: phytotherapy, that didn't work, and Primpéran. During our interview, he was eating candies, got an upset stomach and was afraid he was going to vomit. He was also chewing a liquorice stick, breathing heavily, complained of nausea and had a tense jaw. He spoke to us of changes in his professional environment: new team, intern leaving.

\section{Diagnostic statement}

Agoraphobia without panic disorder (CIM-10: F300.01)

Unspecified mental handicap (CIM-10: F79)

Global Assessment of Functioning: 41-50, serious impairment in social and professional functioning.

\section{Mr. JR's overall understanding of the situation}

The prevalence of mental health disorders, especially affective and anxiety disorders, in people with I.D. reaches $40 \%[11,12]$. Mental health disorders can arise from a multitude of factors which influence each other (biological, psychological, sociological and/or environmental). Initial clinical evaluation and elaborated functional analysis ${ }^{1}$ with Mr. JR conducted the intervention, showing us that the causes of his current difficulties were psychological, meaning both cognitive and affective.

A contract was established with Mr. JR: a BCT contract of 10 sessions to be evaluated ${ }^{2}$. Three therapeutic axes were proposed: work on physiological reactions toward anxiety and the train phobia involving techniques to manage these reactions, cognitive restructuring work, and work on the behaviour with exposure to the anxiety-inducing situation. An antidepressant medication and an anti-anxiety medication were initiated (Table 1).

${ }^{1}$ See SECCA table in the Annexure to this document.

${ }^{2}$ Therapy usually involves several phases: clinical diagnosis and functional analysis; evaluation interviews (using scales and questionnaires); information to the patient about the diagnosis, design and establishment of a therapeutic contract; therapeutic intervention; evaluation of results and end of therapy.

Table 1 Model and therapeutic approach.

\begin{tabular}{|c|c|c|}
\hline $\begin{array}{l}\text { P : } \\
\text { Physically stressed? }\end{array}$ & $\begin{array}{l}\text { Identification of } \\
\text { physical symptoms } \\
\text { linked to anxiety } \\
\text { Obtain information } \\
\text { about anxiety- } \\
\text { inducing situations } \\
\text { Hierarchy of } \\
\text { anxiety-inducing } \\
\text { situations }\end{array}$ & $\begin{array}{l}\text { Review of physical } \\
\text { symptoms linked to } \\
\text { anxiety } \\
\text { Teaching and practice } \\
\text { of relaxation techniques } \\
\text { (square breathing and } \\
\text { progressive muscular } \\
\text { relaxation) }\end{array}$ \\
\hline $\begin{array}{l}\text { E: } \\
\text { Entrenched in negative } \\
\text { thoughts? }\end{array}$ & $\begin{array}{l}\text { Identification of } \\
\text { negative thoughts } \\
\text { in anxiety-inducing } \\
\text { situation }\end{array}$ & Cognitive restructuring \\
\hline $\begin{array}{l}\text { U: } \\
\text { Uncover an attitude or } \\
\text { action to help oneself? }\end{array}$ & $\begin{array}{l}\text { Introduction of } \\
\text { stages for problem- } \\
\text { solving }\end{array}$ & Set-up alternatives \\
\hline $\begin{array}{l}\text { R: } \\
\text { Results and rewards }\end{array}$ & $\begin{array}{l}\text { Introduction of } \\
\text { self-evaluation and } \\
\text { self-reward ideas }\end{array}$ & $\begin{array}{l}\text { Imagined and real-life } \\
\text { exposure to anxiety- } \\
\text { inducing situations }\end{array}$ \\
\hline
\end{tabular}




\section{Model and Therapeutic Approach}

\section{The " PEUR » Intervention plan}

\section{Asking parents and peers}

Information on anxiety and maintenance factors is given to parents and peers in order to normalize the anxiety [13].

\section{Information on the disorder - Explanation and dedramatisation}

Pertinent information about his disorder was given to Mr. JR The prevalence of this disorder in the overall population reassures him that he does not have a serious organic illness ${ }^{3}$. We explain the mechanism of this disorder to him, the physiology of the reactions of fear and anxiety, as well as their function (protection and vigilance). Mr. JR also receives explanations about how his phobia spirals with the three dimensions (physical, cognitive and behavioural), how they interact and self-sustain in a vicious circle (Cognitive Model of the PA) [14]

1. Anxiety is a part of life -2 . Anxiety is not an enemy, one has to know how to accept it and master it -3 . Panic

attacks are a frequent occurrence, even for those who do not have severe psychological problems -4 . The physical symptoms experienced during a panic attack correspond to a cardiac and respiratory acceleration, and not to a heart attack or a stroke -5 . Tension in the intercostal muscles can explain the chest pain often interpreted as an angina pectoris - 6 . The cardiac and respiratory acceleration modifies the gases in the blood (alkalosis). These metabolic changes explain the psychological symptoms of the anxiety which are not caused by the psychological disorder - 7 . These symptoms, which are sometimes called "spasmophilia" or hyperventilation syndrome, can be reproduced in most people by voluntary respiratory acceleration lasting two or three minutes. 8 . The anxiety can be increased by certain catastrophic thoughts or because of unsettling events (Figure 1).

Two of the decisive factors in the success of this therapy with Mr. $J R$ were his motivation to get beyond this, as well as his abilities to understand and conceptualize.

He immediately focused on the fact that his erroneous interpretation (cognitive dimension) in the anxiety-inducing situation came from his perception of the physical signs (interpreted as a threat), inducing an avoidance and running

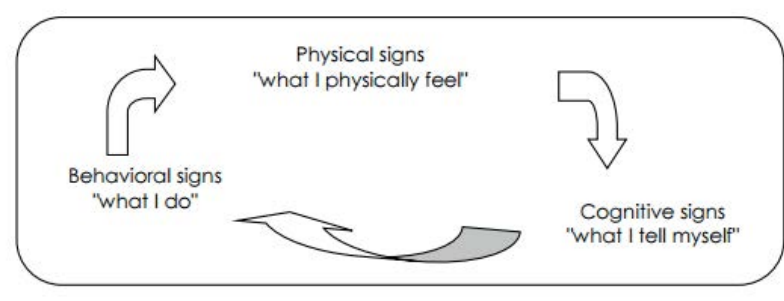

Figure 1 Behavioural signs.

${ }^{3}$ Cottraux (2001) and Cottraux (1995) suggest that during this information phase the following 8 main messages be provided away behaviour, which then lowered his self-esteem and he lost control.

Our priority was to work first on regulating and controlling his respiration (square breathing: breathe in for a count of 4, hold the breath for a count of 4 , breathe out for a count of 4 and wait for a count of 4). This technique helped Mr. JR decrease and prevent the physiological reactions caused by hyperventilating. Relaxation training (according to Schultz's "autogenic training"[15]) enabled him to increase his self-control with respect to anxiety in general. This technique is associated with cognitive restructuration, and made it possible for Mr. J.R. to quickly confront and stop his second phobia (taking the highway in a taxi).

The cognitive intervention made it possible for Mr. JR to interpret his physical signs in a more rational manner. He identified his automatic thoughts and the belief associated with the physical symptoms, and was able to formulate more realistic thoughts.

Following that, we worked on his behavioural reactions using three imagined exposure situations. Because of this motivational approach, Mr. JR asked to take the train again. And this was upon his own initiative and with the help of his parents that he began to travel again without any major problem.

We used an individualized protocol - which was a simple, original and suitable method to promote clinical research. Our protocol consisted of taking the subject as his own witness and evaluating him throughout several phases - both interventional and non interventional.

For the measurements we used a quasi-experimental $A B$ protocol. It included the following three stages, throughout which several behaviours were measured repeatedly.

A baseline which measured the behaviours before any treatment was called T0. Next, a treatment phase was established using a therapeutic procedure and was called T1. The follow-up T2 phase evaluated whether the behavioural changes obtained in T1 were maintained or no longer remained.

For the behavioural measurements, we used Beck's Depression Scale, the Penn State Fears Scale, and the measure of the phobia (train - highway).

By comparing the scores obtained at T0, T2 and T2 which represent the dependent variable-we were able to evaluate the therapeutic intervention.

We opted for three scorings on three scales (Beck's Depression 13 items, Penn State 16 items, Beck's Anxiety inventory 21 items) and in this way had 50 behavioural points on three phrases.

Our procedure was thus largely superior to the scoring required in a quasi-experimental protocol. Indeed, those require a minimum of three behavioural points per phase to analyze the protocol.

\section{Scales and Results:}

1. Depression scale (Beck, 13 items [16]): three measurement - $\mathrm{T} 0-\mathrm{T} 1-\mathrm{T} 2 \rightarrow$ no depression

2. Penn State Fears scale [17]: 16 items, three measurements - $\mathrm{T0} \rightarrow 48$ 
- $\mathrm{T} 1$ (3 months) treatment phase $\rightarrow 25$

- T2 (1 year later) follow-up phase $\rightarrow 28$

3. Beck's anxiety inventory: 21 items, three measurements with extractions of the following items: (Table 2)

4. Global Assessment of Functioning, three measurements

- T0 $\rightarrow 41$ - 50 serious impairment in social and professional functioning.

- T1 (3 months) treatment phase $\rightarrow 41-50$ serious impairment in social and professional functioning.

- T2 (1 year after) follow-up phase $\rightarrow 61$ - 70 some difficulty in social and professional functioning, but generally functioning pretty well, has some positive interpersonal relationships.

The results obtained show that during the baseline phase, the patient does not suffer from depression, but the Penn State Fears questionnaire revealed a mild anxiety disorder accompanied by serious physiological symptoms (Figure 2).

The Global Assessment of Functioning showed a serious impairment in social and professional functioning which manifested itself by a great dependency on the patient's entourage as well as by a retreat from habitual daily activities and leisure activities.

The treatment extended across three months, occurring once a week. The functional analysis demonstrated three therapeutic axes. Work on physiological reactions of anxiety and the train phobia using techniques to manage these reactions; cognitive restructuring work and work on the behaviour with exposure to the anxiety-inducing situation. This work made it possible to reverse the tendency toward an increase of anxiety.

Thanks to the last evaluation, at one year following the treatment (non treatment phase), we were able to report that the improvement obtained in T1 was not a placebo effect, meaning that it was not linked to the patient's positive expectations or a spontaneous positive evolution. It was the stabilization of the results obtained between the intervention and the nonintervention which enabled us to prove the effectiveness of the therapeutic intervention.

Table 2 Beck's anxiety inventory.

\begin{tabular}{|c|c|c|c|}
\hline $\begin{array}{l}\text { Beck's anxiety } \\
\text { inventory: }\end{array}$ & \multicolumn{3}{|c|}{ Degree to which affected } \\
\hline Items & TO & $\begin{array}{c}\mathrm{T} 1 \\
\text { treatment } \\
\text { phase }\end{array}$ & $\begin{array}{c}\mathrm{T} 2 \\
\text { follow-up phase }\end{array}$ \\
\hline $\begin{array}{l}\text { 3. "Weak legs", } \\
\text { trembling in the legs } \\
\text { 4. Incapacity to relax } \\
\text { 7. Marked heartbeats } \\
\text { 17. Feeling of fear }\end{array}$ & $\begin{array}{l}\text { Severely } \\
\text { Severely } \\
\text { Severely } \\
\text { Severely }\end{array}$ & $\begin{array}{l}\text { Not at all } \\
\text { Not at all } \\
\text { Not at all } \\
\text { Not at all }\end{array}$ & $\begin{array}{l}\text { Not at all } \\
\text { Not at all } \\
\text { Not at all } \\
\text { Not at all }\end{array}$ \\
\hline $\begin{array}{l}\text { 5. Fear that the worst is } \\
\text { coming }\end{array}$ & Moderately & Not at all & Not at all \\
\hline 10. Nervousness & Little & Not at all & Not at all \\
\hline
\end{tabular}

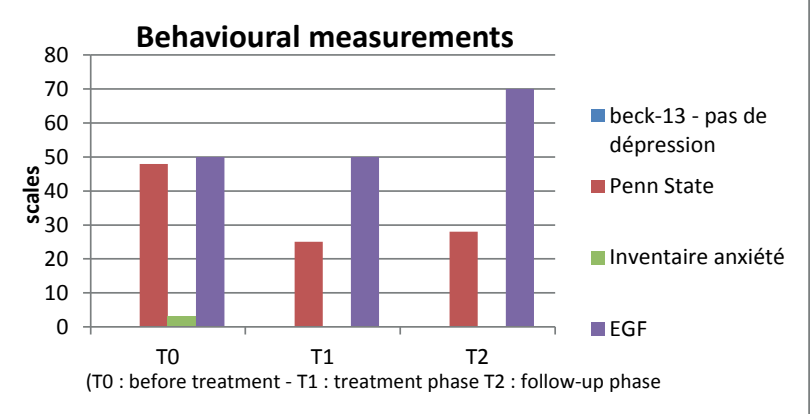

Figure 2

Behavioural measurement.

And for the closely-scheduled and regular measurements, we used the number of blockages per week, as well as the number of times medication was taken. The intensity of the anxiety for the train phobia was also measured and rapidly and significantly lowered.

\section{Conclusion and Discussion}

In conclusion, the therapeutic alliance with Mr. JR his availability and his engagement in the cognitive-behavioural therapeutic process, all worked to rapidly resolve the most difficult situation for him, which had also reduced his autonomy, his self-image and his self-control.

The psycho-educational approach helped Mr. JR understand the phenomena triggering his difficulties and their consequences.

Two other statements resulted from this:

1. BCT works very well with individuals living in a handicap situation. This has already been shown by different authors [18-23].

2. The "PEUR" interventional model seems promising.

The limitation of this individual case study remains the generalization of the therapeutic procedure as well as their conclusion with a larger population living in a situation of mental handicap. Nevertheless, this presentation of a unique case represents, above all, a simple and cost-effective method available for clinicians to function in an experimental way. Indeed, we were able to select measurements, observe and record clinical events onto graphs, which were until now intuitive. In this way, the effectiveness of the behavioural measurements $[17,24-26]$ was demonstrated.

Making this a necessary prelude for any controlled study, but which is personalisable to the individual and the specifics of the mental handicap and which can generate hypotheses that may not appear in group studies. 


\section{References}

1 Essau CA, Conradt J, Petermann F (2000) Frequency comorbidity and psychosocial impairment of anxiety disorders in German adolescents. J Anxiety Disord 14: 263-79.

2 Essau CA, Conradt J, Petermann F (2000) Frequency comorbidity and psychosocial impairment of specific phobia in adolescents. $\mathrm{J}$ Clin Child Psychol N 29: 221-31.

3 Essau CA (2003) Comorbidity of anxiety disorders in adolescents. Depress Anxiety 8: 1-6.

4 Dekker MC, Koot HM (2003) DSM-IV disorders in children with borderline to moderate intellectual disability. II: child and family predictors. J Am Acad Child Adolesc Psychiatry 42: 923-31.

5 Dekker MC, Koot HM (2003) DSM-IV disorders in children with borderline to moderate intellectual disability. I: prevalence and impact. J Am Acad Child Adolesc Psychiatry 42: 915-22.

6 Connor DF, Posever TA (1998) Mental Health Aspercts of Developmental Disabilities 1: 93-102.

7 Wallander JL, Dekker MC, Koot HM (2006) Risk factors for psychopathology in children with intellectual disability: a prospective longitudinal population-based study. J Intellect Disabil Res 50: 259-68.

8 Ollendick TH, Oswald DP, Ollendick DG (1993) Anxiety disorders in mentally retarded persons, in Psychopathology in the mentally retarded, Johnny Matson and R. Barrett, Editors p: 41-85.

9 Stavrakaki C (1999) Depression anxiety and adjustment disorders in people with developmental disabilities, in Psychiatry and behavioural disorders in developmental disabilities and mental retardation, N Bouras, Editor. 1999, Cambridge University Press: Cambridge P : 175-187.

10 Lindsay WR (1999) Treatment of adolescent sex offenders with intellectual disabilities. Ment Retard 37: 201-11.

11 Rojahn J (2003) The Aberrant Behavior Checklist and the Behavior Problems Inventory: convergent and divergent validity. Res Dev Disabil 24: 391-404.

12 Rojahn J, Tassé MJ (1996) Psychopathology in mental retardation, in Manual of diagnosis and professional practice in mental retardation, JW Jacobson and JA Mujick, Editors. APA: Washington p: 147-156.

13 Provencher K (2009) Programme d'intervention cognitivocomportemental auprès d'un groupe d'adolescents anxieux présentant une déficience intellectulle légère à moyenne, in Ecole de psychoéducation, Faculté des artes et des sciences. Université de Montréal: Montréal p: 105.

14 Clark DM, Salkovskis PM, Chalkley AJ (1985) Respiratory control as a treatment for panic attacks. J Behav Ther Exp Psychiatry 16: 23-30.

15 Schultz JH (2013) Le training autogène. PUF Paris.

16 Beck AT (1988) An inventory for measuring clinical anxiety: psychometric properties. J Consult Clin Psychol 56: 893-7.

17 Meyer TJ (1990) Development and validation of the Penn State Worry Questionnaire. Behav Res Ther 28: 487-95.

18 Giuliani FP, El Korh (2015) Case study: the contribution of the neurosciences in the treatment of the anxiety disorders at two people living with autism spectrum disorders and intellectual disabilities. RFCCC 10: 8-22.

19 Giuliani F, El Korh P (2016) Is Storytelling Therapy Useful for Children with Autism Spectrum Disorders and Severe Mental Retardation ? Advanced Techniques in Biology \& Medicine 4: 166-201.

20 Giuliani F, El Korh P (2016) Adult with Autistic Spectrum Disorders: Specialized Treatment. Advanced Techniques in Biology \& Medicine 4: 164-8.

21 Giuliani F (2009) Organisation de l'exploration oculaire dans le cadre d'un traitement de l'évitement du contact visuel, in TCC et neurosciences. Elsevier Masson: Issy-les-Moulineaux p: 218.

22 Giuliani F, Perrenoud V, Favrod J (2014) Using eye-tracking as support for the TEACCH program and two teenagers with autism-spectrum disorders. Revue Francophone de Clinique Comportementale et Cognitive, XIX: 39-56.

23 Grasset F (2008) Mental retardation and psychiatry. Rev Med Suisse 4 1976-8.

24 Beck AT (1991) Cognitive therapy. A 30-year retrospective. Am Psychol 46: 368-75.

25 Beck AT, Steer RA (1991) Relationship between the Beck Anxiety Inventory and Hamilton Anxiety Rating Scale with anxious outpatients. Journal of Anxiety Disorders 5: 213-223.

26 Beck AT (1991) Panic disorder and suicidal ideation and behavior: discrepant findings in psychiatric outpatients. Am J Psychiatry 148: 1195-9. 